\title{
Laws of volume elasticity in physical processes of the effect of $T, P, H$ parameters on the lattice modifications, phase transitions, conductivity and magnetism
}

\author{
Petr Polyakov \\ Institute for Physics of Mining Processes, 72 R. Luxemburg Str., 83114 Donetsk
}

\begin{abstract}
Analysis of the experimental results allows to single out the law of bulk elasticity that implies that the structural binding energy is formed by physical and chemical methods and transformed into elastic stresses that control lattice changes, formation of phase transitions, resistivity and magnetism in the course of physical processes under the effect of parameters. The laws of volume thermo-, baro- and magnetoelasticity are the determining factors in lattice modifications in the structure sites together with the composition variation.
\end{abstract}

\section{Introduction}

The laws of volume thermo-, baro- and magnetoelasticity are the determining factors in lattice modifications in the structure sites together with the composition variation. It is the binding energy transformed into the stresses due to thermoelastic compression that redistributes the state of electronphonon bonds, the volume and the density. Besides, baro- and magnetoelasticity combined with magnetic uncompensation demonstrate "cooling" and "heating" effects due to additional energy of strains that allow redistribution of free and semi-free electrons into a phonon bond and adding to modification of the structure as a whole. The deformation factor accompanied by thermoelastic strains determining phase transitions and magnetic field uncompensation control regularities of conductivity jump in the form of colossal magnetoresistance. The presented work is aimed at settling the question of the presence of anomalies and regularities in view of the laws of the bulk elasticity.

\section{Results and discussion}

When analyzing a wide spectrum of the results of the experimental studies of physical processes under the thermodynamic parameters varied, a question arises, if an analogy in the effects should be singled out. A number of known scientists contributed to the investigation of the magnetic field effect as presented in the Kapitsa's works [1]. The experiments were based on the employment of temperature and high magnetic fields. The works of Bridgeman [2] dealt with a wide pressure range at a fixed temperature. In Vereschagin's works [3], a number of methods involving high pressure were

\footnotetext{
* Corresponding author: poljakov@mail.fti.ac.donetsk.ua
}

presented. The works of A.A. Galkin scientific school [4] were very representative, because they employed sensitive methods of investigation and extensive results about the effect of three parameters were analyzed.

The main determining factor of the evolution in the form of deforming elastic stress is the elastic energy that is higher of the energies of other microprocesses. This fact requires understanding of the interaction laws within the structure, to control modifications of the density, volume, the number of the neighbors, alternation of the high symmetry to the low one (polymorphous transformations related to the reconstruction of the structure as a transition to a dense packed structure). Phase transformations are associated with a volume jump and insignificant structure transformation within the range of $1 \%$, with the anisotropy of the elastic stresses participating. It should be noted that the energy of the elastic stresses is the basis separating the phase states of the physical processes [5].

Under the effect of $\mathrm{H}$ and $\mathrm{P}$, energy changes are generated in the structure sites of the samples. The changes of the energy affect the state of the electron and phonon subsystems that are analogous to those caused by impurities or defects.

X-ray methods provided registered four-fold difference in the pressure effect on the structure compressibility that emphasizes the role of the anisotropy of elasticity [6]. The analysis of the effect of magnetoelastic and elastic energies allowed separation of combinations of the parameters of the elastic interactions. The results of the studies of magnetcontaining semiconductors revealed a regularity in the $\mathrm{T}$ $\mathrm{H}-\mathrm{P}$ effect [7]. It was found that the effect of $6,2 \mathrm{~K}$ corresponds to that of 2,7 kOe and $1 \mathrm{kbar}$ [8]. This was an evidence of the causing role of the structure modifications. 
Analogies and regularities of the phase state modifications were established in single crystal semiconductors and dielectrics. The presented results illustrate the causing role of the deforming elastic stresses that modifies the structure and the properties and forms the basis of the mechanics in the course of the structural transformations in a wide spectrum of solids.

Further, an attention is concentrated on the analysis of the studies of manganite-based polycrystal structures and cuprate-based monocrystals [5]. As examples, the measurements of conductivity [9] are presented; the temperature dependence under the effect of the hydrostatic pressure $\mathrm{P}$ and the magnetic field $\mathrm{H}$ is separated. Some details of the reduction of the resistance within the whole temperature range are observed. The metal part of the resistance reduction is explicitly emphasized to compare it with Fig.1. As a comparison, Fig.2 is presented [10] where the resistance of the $\mathrm{La}_{1-x}$ $\mathrm{Sr}_{\mathrm{x}} \mathrm{MnO}_{3}$ samples is modified in the course of the processes of varied composition $x$. Thus, any structural changes are reflected in the properties and these are the laws of the volume elasticity that are the determining factors of the measurements in the physical processes of the parameter effects.

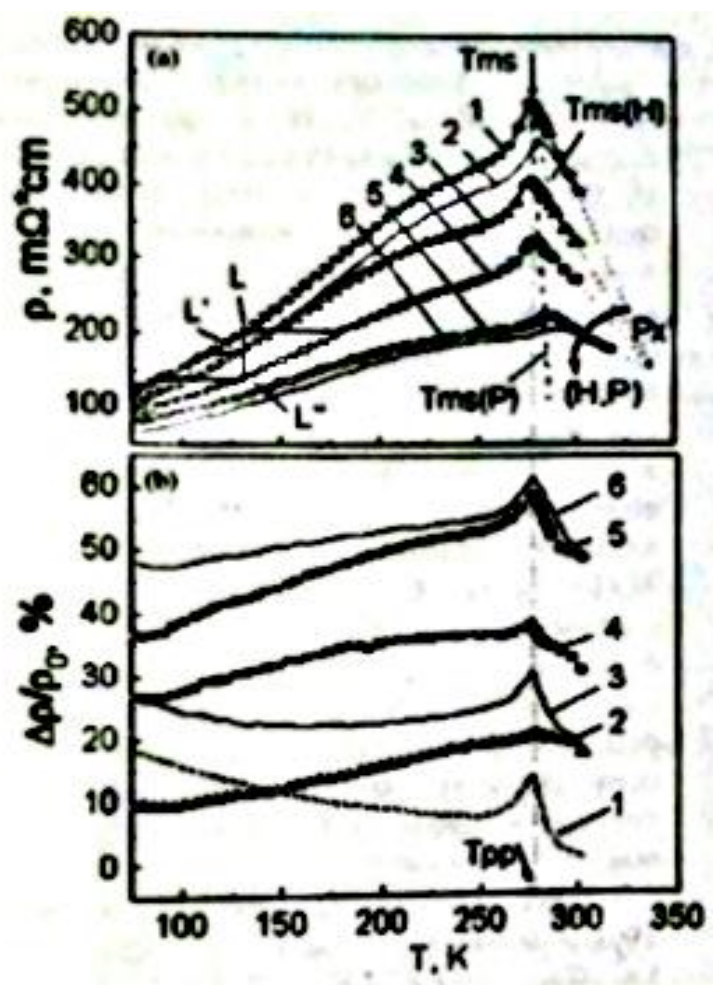

Fig.1. Temperature dependence of the resistivity (a) and magneto-, baro-, magnetobaroresistive effects (b) in $\mathrm{La}_{0.7} \mathrm{Ca}_{0.3} \mathrm{MnO}_{3}$ under the effect of magneretic field and hydrostatic pressure: a) $1-\mathrm{P}=0, \mathrm{H}=0 ; 2-\mathrm{P}=0, \mathrm{H}=8 \mathrm{kOe}$; $3-\mathrm{P}=6$ kbar, $\mathrm{H}=0 ; 4-\mathrm{P}=12 \mathrm{kbar}, \mathrm{H}=0 ; \mathrm{P}=17$ kbar, $\mathrm{H}=0$; b) $1-\mathrm{P}-0, \mathrm{H}=8 \mathrm{kOe} ; 2-\mathrm{P}=6 \mathrm{kbar}, \mathrm{H}=0 ; 3-\mathrm{P}=6$ kbar, $\mathrm{H}=8$ kOe; $4-\mathrm{P}=12$ kbar, $\mathrm{H}=0$; $5-\mathrm{P}=17$ kbar, $\mathrm{H}=0$; $6-\mathrm{P}=17$ kbar, $\mathrm{H}=8 \mathrm{kOe}[5]$.

The introduced energy of the elastic stresses is transformed into the united energy that forms electron and phonon bonds. The energy of $0.1 \mathrm{eV}$ corresponds to
$10 \mathrm{kbar}$. In the course of the structure formation, the binding energy of the nucleus and the electrons of the closed internal shells is much higher of the binding energy of the stressed shells. These are the energies that play the major role in the formation of the site structure and redistribution of the electron levels in the phonon structure. In the course of gradual filling of the external shells, an additional energy of the elastic stresses related to the binding energy is about $0,1-1 \mathrm{eV}$.

The selected research area including the volume elasticity allows establishing the regularities of the elasticity anisotropy in a magnetodielectric [11]. The regularities of the first-order phase transition have been found in the frequency-field dependences in three parameters. The temperature of the phase transition at $9,8^{\circ} \mathrm{K}[12]$ has been demonstrated first.

Now we shall evaluate the levels of energy contribution in the course of consideration of critical phenomena and properties. This matter touches upon principal questions of the direct relation between thermodynamic parameters and structure evolution through the mechanisms of elastic stresses affecting the volume and other parameters controlling the structural phase transitions and the properties of phase states. We shall estimate the energy of Coulomb interactions as $1 \div 10 \mathrm{eV}$, the influence of the crystal field as $0.1 \div 1 \mathrm{eV}$, spin-orbit relationship as $10^{-2} \div 10^{-1} \mathrm{eV}$, spin-spin (magnetic) bond as $10^{-4} \mathrm{eV}$, electron-nuclear bond as $10^{-4} \div 10^{-5} \mathrm{eV}$. According to our estimations, the energy of elastic stresses with taking into account the coefficient of compressibility is $1 \div 10 \mathrm{eV}$.

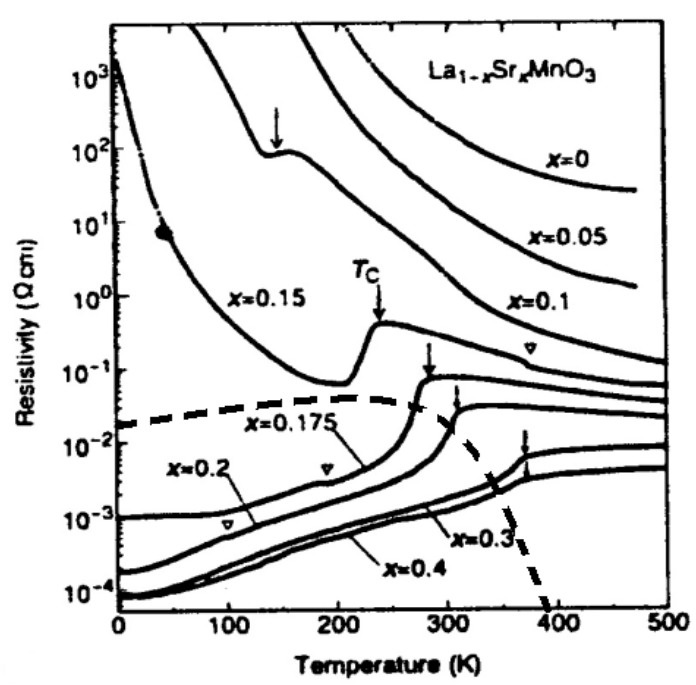

Fig.2. Temperature dependence of the resistivity for various single crystals of $\mathrm{La}_{1-\mathrm{x}} \mathrm{Sr}_{\mathrm{x}} \mathrm{MnO}_{3}$. Arrows indicate the Curie temperature. The open triangles indicate anomalies due to structural transitions [10].

Unfortunately, methods of investigations under the hydrostatic pressure were far from being perfect at that time. Our result shows only a combined T-P-H effect (effect of temperature, pressure and magnetic field) through mechanisms of elastic deforming (ED) stresses. It provides an objective consideration of physical 
processes; otherwise, new approaches cannot be elaborated. The magnetism of magnet-containing structures can get the most effective consideration from the viewpoint of the experiment. There the real physical process is registered if the form of the evolution of the properties of a solid that testifies the regular bond of the changes of the crystal structure and the electronic and magnetic properties.

Magnetization is a consequence of the influence of the magnetic field on magnetically non-compensated constituent of a particle provided it is high enough to affect the structure significantly.

\section{Conclusions}

We should take into account that quantum mechanical forces forming the magnetism are of short range in fact, so deep understanding of the regularity if interaction is necessary for the estimation of this process. The elastic energy is an immediate energy of long range. These are elastic stresses in the structure that form the linearity of the magnetization regardless of important details of microscopic interactions. By definition, atoms and molecules have small magnetic moments of noncompensation. The structure is the totality of molecules and atoms brought into the sites of the structure (several atoms and molecules) and bound in the lattice by the compatibility of valent and free electrons. The state of the structure and its predisposition to paramagnetism is formed by high-temperature physical and chemical processes where the magnetic factor is absent by definition. In other words, the high-temperature area has no ability for magnetization.

The succeeding cooling and bulk compression result in the loss of heat energy transformed into the loss of the energy of the elastic stresses in the structure. This process is followed by the linear evolution of the parameter due to the mechanisms of bulk elasticity.

The object of the present paper is an analysis, drawing analogies and generalization of regularities of an evolution of structural phase transitions and properties in different multi-component magnetcontaining structures. It is also aimed at the presentation of unambiguous T-P-H impact through mechanisms of elastic anisotropic deforming stresses and settling the question of the presence of anomalies and regularities in view of the laws of the bulk elasticity.

The presented results allow statement that the basis of magnetism is formed in the energetic bonds of the structure sites. Here magnetic non-compensation is manifested that appears in the course of electron-phonon interaction under the effect of the introduced energies to be transformed into the elastic stresses by the effect of temperature, pressure and magnetic field.

\section{References}

1. P.L. Kapitsa, The strong magnetic fields, Nauka, Moscow, 1988 (in Russian).
2. P. Bridgmen, Newest works in the high-pressure area, Nauka, Moscow, 1948 (in Russian).

3. L.F. Vereschagin, Solids under high pressure, Nauka, Moscow., 1981 (in Russian).

4. A.A. Galkin,, Physics of Solid State, Naukova dumka, Kiev, 1986 (in Russian).

5. P.I. Polyakov, T.A. Ryumshina, Magnetism and laws of bulk elasticity, Transworld research network, Kerala, 2009.

6. A.A. Galkin, S.V. Ivanova, P.I. Polyakov, V.I. Kamenev, Structural, magnetic and resonance properties of $\mathrm{CuCl}_{2} 2 \mathrm{D}_{2} \mathrm{O}$ Sol. State Phys., 8 (1979) 2580-2583

7. P.I. Polyakov, S.S. Kucherenko, Regularities in temperature, magnetic field and pressure effect on the resistive properties of magnetic semiconductors, JMMM 248, (2002) 396-401.

8. P.I. Polyakov, S.S. Kucherenko, Elastic deformation mechanisms of the effect of temperature, magnetic field and pressure on the resistive and magnetic properties of magnetic semiconductors, Fiz. Nizk.Temp. 28, (2002) 1041-1047.

9. P.I Polyakov, S.S. Kucherenko, Dynamics of structural phase transition and changes in properties under the influence of elastically anisotropic deforming stresses, regularities of critical lines and points in magnetic semiconductors and magnetic dielectrics, JMMM 278, (2004) 138-155.

10. E. Dagotto, T. Hotta, A.Moreo, Colossal magnetoresistant materials: the key role of phase separation, Phys. Rep. 344, (2001) 1-153.

11. A.A Galkin, S.V. Ivanova, V.I. Kamenev, Magnetyostriction effect on resonant properties of cuprpus chloride dehydrate under pressure, Proc. Of XX congress AMPERE, Tallinn, 1978.

12. P.I Polyakov, Materials science Feb.2015 arxiv: 1502.07473 